\title{
Method for Generating Long-Range Correlations for Large Systems
}

\author{
Hernán A. Makse ${ }^{(a)}$, Shlomo Havlin ${ }^{(a),(b)}$, Moshe Schwartz $^{(b)}$ and H. Eugene Stanley ${ }^{(a)}$ \\ (a) Center for Polymer Studies and Department of Physics, Boston University, Boston, Massachussetts 02215 \\ (b) Department of Physics, Bar-Ilan University, Ramat-Gan, ISRAEL
}

(October 17, 2018)

\begin{abstract}
We propose a new method to generate a sequence of random numbers with long-range powerlaw correlations that overcomes known difficulties associated with large systems. The new method presents an improvement on the commonly-used methods. We apply the algorithm to generate enhanced diffusion, isotropic and anisotropic self-affine surfaces, and isotropic and anisotropic correlated percolation.
\end{abstract}

PACS numbers:

\section{INTRODUCTION}

Recently, the study of physical systems displaying long-range power-law correlation has attracted considerable attention. Long-range correlations have been found in a wide number of systems including biological, physical, economical, geological and urban systems [1]. Attempts to study and characterize such systems are often based on numerical methods to generate correlated noise [2.3]. One of the most used methods to generate a sequence of random numbers with power-law correlations is the Fourier filtering method (Ffm) [2, 1, D]. It consists of filtering the Fourier components of a uncorrelated sequence of random numbers with a suitable power-law filter in order to introduce correlations among the variables. This method has the disadvantage of presenting a finite cutoff in the range over which the variables are actually correlated [4 [6]. As a consequence, one must generate a very large sequence of numbers, and then use only the small fraction of them that are actually correlated (this fraction can be as small as $0.1 \%$ of the initial length of the sequence [. [. 5 ). This limitation makes the Ffm not suitable for the study of scaling properties in the limit of large systems.

Here we modify the Ffm in order to remove the cutoff in the range of correlations. We show that in the modified method the actual correlations extend to the whole system. We also apply the method to generate several systems such as fractional Brownian motion, self-affine surfaces and long-range correlated percolation.

\section{FOURIER FILTERING METHOD}

We start by defining the Ffm [2, 4, 5] for the $d=1$ case ( $d$ is the dimension of the sample). Consider a stationary sequence of $L$ uncorrelated random numbers $\left\{u_{i}\right\}_{i=1, \ldots, L}$. The correlation function is $\left\langle u_{i} u_{i+\ell}\right\rangle \sim \delta_{\ell, 0}$, with $\delta_{\ell, 0}$ the Kronecker delta, and the brackets denote an average with respect to a Gaussian distribution. The goal is to use the sequence $\left\{u_{i}\right\}$, in order to generate a new sequence, $\left\{\eta_{i}\right\}$, with a long-range power-law correlation function $C(\ell)$ of the form

$$
C(\ell) \equiv\left\langle\eta_{i} \eta_{i+\ell}\right\rangle \sim \ell^{-\gamma} \quad[\ell \rightarrow \infty] .
$$

Here, $\gamma$ is the correlation exponent, and the long-range correlations are relevant for $0<\gamma<d$, where $d=1$. The spectral density $S(q)$ defined as the Fourier transform of $C(\ell)$ [7] has the asymptotic form

$$
S(q)=\left\langle\eta_{q} \eta_{-q}\right\rangle \sim q^{\gamma-1} \quad[q \rightarrow 0] .
$$

Here $\left\{\eta_{q}\right\}$ correspond to the Fourier transform coefficients of $\left\{\eta_{j}\right\}$, and satisfies

$$
\eta_{q}=(S(q))^{1 / 2} u_{q},
$$

where $\left\{u_{q}\right\}$ are the Fourier transform coefficients of $\left\{u_{i}\right\}$.

The actual numerical algorithm for Ffm consists of the following steps:

(i) Generate a one-dimensional sequence $\left\{u_{i}\right\}$ of uncorrelated random numbers with a Gaussian distribution, and calculate the Fourier transform coefficients $\left\{u_{q}\right\}$ [ [ $]$.

(ii) Obtain $\left\{\eta_{q}\right\}$ using (2) and (3).

(iii) Calculate the inverse Fourier transform of $\left\{\eta_{q}\right\}$ to obtain $\left\{\eta_{i}\right\}$, the sequence in real space with the desired power-law correlation function (11).

\section{PRESENT METHOD}

The Ffm method has been applied in a number of studies of correlated systems [1, [, , , ]. However, an analysis of the method for large $L$ shows that, by following the above procedure, one ends up with a sequence of correlated numbers whose range of correlations, for $d=1$, is only about $0.1 \%$ of the system size. For example, from an initial sequence of $10^{6}$ numbers, only $10^{3}$ numbers show the desired power-law correlations 海. For $d=2$, the range of correlations increases to $1 \%$ of the system size [5]. In order to remove this artificial cutoff in the correlations, we modify the Ffm algorithm as follows:

(a) To calculate the spectral density $S(q)$, a welldefined correlation function in the real space is needed. The function $C(\ell)=\ell^{-\gamma}$ has a singularity at $\ell=0$. We 
replace (11) with a slightly modified correlation function that has the desired power-law behavior for large $\ell$, and is well-defined at the origin

$$
C(\ell) \equiv\left(1+\ell^{2}\right)^{-\gamma / 2}
$$

(b) The relation (3) is based on the convolution theorem, and therefore the desired correlation function (4) must satisfy the proper periodic boundary condition. The function $C(\ell)$ can be naturally extended to negative values of $\ell$ due to the $\ell^{2}$ dependence. We define (14) in the interval $[-L / 2, \ldots, L / 2]$, and impose periodic boundary conditions, i.e. $C(\ell)=C(\ell+L)$.

(c) The discrete Fourier transform of (4) - needed to obtain $\eta_{q}$ using (3) - can now be calculated analytically,

$$
S(q)=\frac{2 \pi^{1 / 2}}{\Gamma(\beta+1)}\left(\frac{q}{2}\right)^{\beta} K_{\beta}(q),
$$

where $q$ takes values $q=2 \pi m / L$ with $m=-L / 2, \ldots, L / 2$, $K_{\beta}(q)$ is the modified Bessel function of order $\beta=$ $(\gamma-1) / 2$, and $\Gamma$ is the Gamma function.

The modified Bessel functions satisfy the asymptotic relations

$$
K_{\beta}(q)= \begin{cases}\frac{\Gamma(\beta)}{2}\left(\frac{q}{2}\right)^{\beta} & \text { if } q \ll 1 \\ \sqrt{\frac{\pi}{2 q}} e^{-q} & \text { if } q \gg 1\end{cases}
$$

for $\beta$ positive and by definition $K_{-\beta}=K_{\beta}$. Then for small values of $q$, (5) gives the same asymptotic form as (2). However, the Bessel function introduces a cutoff for large $q$ in the sense that $S(q)$ has a faster exponential decay. This cutoff, while irrelevant to the longdistance scaling, is very important for the validity of the whole Fourier analysis because it avoids aliasing effects (see Chapter 7 in [9]). The cutoff in the Fourier space is thus responsible for eliminating the cutoff in real space observed in the Ffm method.

In order to perform the above steps numerically, we employ the Fast Fourier transform [9,10]. Due to the periodic boundary condition imposed on the correlation function, it follows that the correlated sample satisfies the same periodicity. If one requires a sequence with open boundary conditions, we generate twice as many numbers and then split the sequence in two parts [11].

To test the actual correlations of the generated sample $\left\{\eta_{i}\right\}$ we calculate $C(\ell)$ averaging over different realizations of random numbers. Figure 1 shows a plot of the actual correlations obtained for different values of $\gamma$ and for a sequence of $L=2^{21}$ numbers. It is seen that the long-range correlations exist for the whole system. The nominal values of $\gamma$ obtained from the best fits are also the same, within the error bars, as the desired input values.

To summarize the method, the correlation function we propose is well-defined and satisfies the correct powerlaw behavior in the real space. Its Fourier transform has the correct power law at small frequencies, and presents a cutoff for large frequencies that avoids aliasing effects, and leads to the infinite long-range behavior in real space. An alternative method in which $S(q)$ was calculated numericaly, in contrast to the analytical expression (5), is given in [12].

\section{APPLICATIONS}

In the following we apply the proposed method to several physical problems.

\section{A. Generating fractional Brownian motion (fBm).}

We map the variables $\left\{\eta_{i}\right\}$ onto the steps of a correlated random walk, and define the position of the walker at step $t$ by $x(t)=\sum_{i=1}^{t} \eta_{i}$. Then, $x(t)$ corresponds to a $t$-step fBm, and the sequence of increments $\left\{\eta_{i}\right\}$ is called fractional Gaussian noise (fGn) [13]. An important quantity is the mean-square displacement of the $\mathrm{fBm}$ whose asymptotic behavior is

$$
\left\langle\left|x(t)-x\left(t_{0}\right)\right|^{2}\right\rangle \sim\left|t-t_{0}\right|^{2-\gamma}
$$

Thus the long-range correlations lead to enhanced diffusion [14] $\left\langle\left|x(t)-x\left(t_{0}\right)\right|^{2}\right\rangle \sim\left|t-t_{0}\right|^{2 H}$ for $0<\gamma<1$ with $H=1-\gamma / 2[15]$. Figure 2 shows the plots of the mean square displacement for different degree of correlations. The fits confirm the validity of the long range correlations among the variables in the whole system size.

\section{B. Generating long-range correlations in $d$ dimensions.}

The algorithm can be easily generalized to higher dimensions. In a $d$-dimensional cube of volume $L^{d}$ the desired correlation function takes the form

$$
C(\vec{\ell})=\left(1+\sum_{i=1}^{d} \ell_{i}^{2}\right)^{-\gamma / 2}
$$

with the corresponding periodic boundary condition, $C(\vec{\ell})=C(\vec{\ell}+\vec{L})$. The spectral density is

$$
S(\vec{q})=\frac{2 \pi^{d / 2}}{\Gamma\left(\beta_{d}+1\right)}\left(\frac{q}{2}\right)^{\beta_{d}} K_{\beta_{d}}(q),
$$

where $q=|\vec{q}|, q_{i}=2 \pi m_{i} / L,-L / 2 \leq m_{i} \leq L / 2$, $i=1, \ldots, d$, and $\beta_{d}=(\gamma-d) / 2$. In the two-dimensional case the correlated variables are defined in a xy square lattice $\left\{\eta_{i, j}\right\}$. Figure 3 shows a test of the actual correlations obtained in two dimensions for different degree of correlations, and for a system of $L=2^{11}$. 


\section{Generating $\mathrm{fBm}$ in two dimensions}

The two-dimensional correlated numbers $\left\{\eta_{i, j}\right\}$ can be used to generate two-dimensional $\mathrm{fBm}$. We propose the following definition 16]

$$
h(t, s) \equiv \sum_{i=1}^{t} \eta_{i, s}+\sum_{j=1}^{s} \eta_{t, j}
$$

After some algebra, we find that when the numbers $\left\{\eta_{i, j}\right\}$ are long-range correlated then

$$
\left\langle\left|h(t, s)-h\left(t_{0}, s_{0}\right)\right|^{2}\right\rangle \sim\left|\left(t-t_{0}\right)^{2}+\left(s-s_{0}\right)^{2}\right|^{1-\gamma / 2}
$$

Thus, using the correlated numbers with $0<\gamma<2$, fBm can be generated with exponent given by $H=1-\gamma / 2$ and $0<H<1$. A landscape with this scaling behavior is also called self-affine surface 117 .

\section{Generating anisotropic long-range correlations}

Many physical systems display not only correlations but also anisotropy [1] reflected in different correlation exponents along different directions. We generalize the algorithm for this case. We propose a correlation function suitable for two-dimensional anisotropic systems

$$
C(r, \varphi)=r^{-\gamma_{x}} \cos ^{2} \varphi+r^{-\gamma_{y}} \sin ^{2} \varphi
$$

where $(r, \varphi)$ are the polar coordinates. The spectral density is

$$
\begin{aligned}
S\left(q, \varphi_{q}\right)= & \frac{\pi^{3 / 2} \Gamma\left(1+\beta_{x} / 2\right)}{2^{1-\beta_{x}} \Gamma\left(2-\beta_{x} / 2\right)} \frac{\cos ^{2} \varphi_{q}}{q^{\beta_{x}}}+ \\
& \frac{\pi^{3 / 2} \Gamma\left(1+\beta_{y} / 2\right)}{2^{1-\beta_{y}} \Gamma\left(2-\beta_{y} / 2\right)} \frac{\sin ^{2} \varphi_{q}}{q^{\beta_{y}}},
\end{aligned}
$$

with $\beta_{x}=2-\gamma_{x}$, and $\beta_{y}=2-\gamma_{y}$. Then the proposed method can be applied to generate anisotropic correlated numbers. This method might be suitable for the simulation of geological reservoir systems for which strong anisotropy is found [1]. Moreover, after generating the anisotropic variables $\eta$, we can apply the procedure outlined in $\mathbf{C}$ in order to obtain an anisotropic self-affine surface.

\section{E. The correlated percolation problem}

A qualitative check of the impact of long-range correlations for physical systems can be obtained by applying the proposed method to a concrete physical problem: the correlated percolation [18]. The properties of long-range correlated site percolation in the square lattice have been recently studied [5]. However, these studies were limited to systems not larger than $104 \times 104$ sites. The method we present here allows us to study this problem in the limit of large systems. Figure 4 illustrates the results obtained for site percolation on a square lattice of $1024 \times 1024$ sites. We see that the introduction of long-range correlations among the occupancy variables strongly affects the morphology of the system. In the correlated case the clusters look more compact than in the uncorrelated case. The lack of correlations in the uncorrelated case is seen from the presence of many small black holes inside the large clusters (Fig. 4d d). Also, at small concentrations there are only small clusters (Fig. Ac) while for the correlated case, large clusters are present even at low concentration (Fig. 4a).

We wish to thank R. Cuerno, P. Jensen, P. R. King C.-K. Peng, S. Prakash, R. Sadr, and S. Tomassone for discussions. We thank K. B. Lauritsen for discussions leading to (10). The authors thank BP and NSF for financial support.

[1] Dynamics of Fractal Surfaces, edited by F. Family and T. Vicsek (World Scientific, Singapore, 1991); M. Sahimi, Rev. Mod. Phys. 65, 1393 (1993); A. Bunde, S. Havlin, eds., Fractals in Science (Springer-Verlag, Berlin 1994); S. Havlin et al. Phys. Rev. Lett. 61, 1438 (1988); H. A. Makse, S. Havlin, and H. E. Stanley, Nature 377, 608 (1995).

[2] D. Saupe in The Science of Fractal Images, H.-O. Peitgen and D. Saupe, eds. (Springer-Verlag, New York 1988); J. Feder, Fractals (Plenum Press, New York 1988).

[3] See e.g. B. B. Mandelbrot, Water Resour. Res. 7, 543 (1971); R. F. Voss in Fundamental Algorithms in Computer Graphics, edited by R. A. Earnshaw (SpringerVerlag, Berlin 1985).

[4] C.-K. Peng et al. Phys. Rev. A 44, 2239 (1991).

[5] S. Prakash et al. ibid 46, R1724 (1992).

[6] Other methods present similar problems. See for instance Chapter 9 in Feder's book [2].

[7] R. Reif, Fundamental Statistical and Thermal Physics (Mc Graw-Hill, 1965).

[8] In practice one can generate directly $\left\{u_{q}\right\}$ from a sequence of uncorrelated random numbers.

[9] W. H. Press, S. A. Teukolsky, W. T. Vetterling, and B. P. Flannery, Numerical Recipes in Fortran, 2nd ed. (Cambridge University Press, Cambridge 1992).

[10] The calculation of the regular Fourier transform involves $O\left(L^{2}\right)$ operations. Using the Fast Fourier transform algorithm [9] the process is computed in $O(L \log L)$ operations, which gives a drastic difference in computing time, and makes this method very fast.

[11] Another numerical detail that should be considered is that the correlation function in the Fourier space in not defined for $q=0$. This comes from the fact that we are using a continuum limit to calculate the Fourier transform instead of the discrete definition. However, the zero frequency only adds an additive constant to the numbers, and does not affect the scaling properties of the sequence. 
This singularity can be avoided anyway, by assigning a suitable numerical value $0<m_{0}<1$ instead of $m=0$.

[12] H. A. Makse, S. Havlin, H. E. Stanley, and M. Schwartz, Proc. 1993 Int. Conf. Complex Systems in Computational Physics, Buenos Aires, Chaos, Solitons and Fractals 6, 295 (1995). Independently, the same method was also published in N.-N. Pang, Y.-K. Yu, and T. Halpin-Healy, Phys. Rev. E, 523224 (1995).

[13] B. B. Mandelbrot and J. W. Van Ness, SIAM Rev. 10, 442 (1968).

[14] M. F. Shlesinger, J. Klafter, Phys. Rev. Lett. 54, 2551 (1985).

[15] Antipersistent behavior $(0<H<1 / 2)$ cannot be obtained using (4). In this case, the correlation function must satisfy $C(\ell) \sim-\ell^{-\gamma}$, for $\ell \rightarrow \infty$, and $\int_{0}^{\infty} C(\ell) d \ell=$ 0 [13.

[16] See also K. B. Lauritsen, Ph.D. thesis, Aarhus University (unpublished, 1994).

[17] T. Vicsek, Fractal Growth Phenomena, 2nd ed. (World Scientific, Singapore 1991).

[18] A. Coniglio, et al. J. Phys. A 10, 205-209 (1977); A. Weinrib, Phys. Rev. B 29, 387-395 (1984).

FIG. 1. A log-log plot of the average correlation $C(\ell)$ of 50 correlated samples obtained with the proposed method for $L=2^{21}$. Shown are results for different values of the desired $\gamma=0.2,0.4,0.6$, and 0.8 (from top to bottom). The dashed lines represent the best fits which yield the nominal values of $\gamma=0.19 \pm 0.02,0.39 \pm 0.02,0.60 \pm 0.03$ and $0.79 \pm 0.03$. The correlations are calculated until $L / 2$ due to the periodic boundary conditions.
FIG. 2. Log-log plot of the mean square displacement for the $\mathrm{fBm}$, for the same values of the desired $\gamma$ as in Fig. 1 (from bottom to top). The slopes of the linear fits yield $2-\gamma=$ $1.78 \pm 0.02,1.60 \pm 0.02,1.42 \pm 0.03$, and $1.23 \pm 0.03$ respectively, in agreement with (7).

FIG. 3. Log-log plot of the correlations along the diagonal direction in a square lattice of $2^{11} \times 2^{11}$. Shown are results for different values of $\gamma=0.4,0.8,1.2$ and 1.6 (from top to bottom), and we take averages over 50 samples. The fits yield nominal values of $\gamma=0.41 \pm 0.02,0.81 \pm 0.03,1.20 \pm 0.03$ and $1.59 \pm 0.04$.

FIG. 4. Site percolation in the square lattice of $1024 \times 1024$ for different degrees of correlations and concentrations. Figures $4 a$ and $4 b$ correspond to the correlated case with $\gamma=0.2$, while Figs. $4 c$ and $4 d$ correspond to the uncorrelated percolation problem, below and at the threshold for both cases, respectively. Unoccupied sites are in black and occupied sites are in white. Figure $4 e$ shows the case of anysotropic percolation generated with the method of $\mathbf{D}$ for $\gamma_{x}=0.2$ and $\gamma_{y}=1.8$, and for the same concentration as in Fig. $4 a$. We notice how the clusters are elongated along the direction of the smaller exponent (strong correlations). The figures are generated using the same seed for the random number generator. 